Thorax, 1980, 35, 351-354

\title{
Hypoplastic left heart syndrome: can this malformation be treated surgically?
}

\author{
SIMCHA MILO, SIEW YEN HO, AND ROBERT H ANDERSON \\ From the Department of Thoracic and Cardiovascular Surgery, The Sheba Medical Centre, Sackler School of \\ Medicine, Tel-Hashomer, Israel, and the Departments of Paediatrics and Surgery, Cardiothoracic Institute, \\ Brompton Hospital, London
}

ABSTRACT A corrective technique for the surgical treatment of hypoplastic left heart syndrome is proposed. This technique is compatible with subsequent growth because it uses the patient's own tissues exclusively.

The hypoplastic left heart syndrome is a rare but lethal congenital malformation. Most infants with this lesion die within the first week or two of life. ${ }^{12}$ Today's advanced diagnostic methods now permit a rapid and accurate anatomical assessment of such cases. With surgical techniques available such as cardiopulmonary bypass, hypothermia, cardioplegia, and total circulatory arrest, it seems reasonable to explore new surgical approaches aimed at re-establishing physiological flows and pressures in these hearts. These may provide a situation which will be compatible with life and enable reasonable growth and development. They could provide the child with an adequate quality of life.

The purpose of this communication is to propose a surgical technique for correction of the malformation of the hypoplastic left heart syndrome. It is not dissimilar from a recent suggestion by Albert and Bryant ${ }^{3}$ for treatment of this condition, but it has the advantage of using the patient's own tissues exclusively and does not require artificial conduits.

\section{Morphology}

The term "hypoplastic left heart syndrome" describes the condition in which aortic atresia or severe stenosis is associated either with absence of the left atrioventricular connexion or else severe mitral hypoplasia. In either case, the chamber of left ventricular morphology is hypoplastic or rudimentary. Usually the ventricular septum is intact, and pulmonary venous return is distributed to the body via the right atrium,

Address for reprint requests: Dr RH Anderson, Cardiothoracic Institute, Brompton Hospital, Fulham Road, London SW3 6HP. right ventricle, pulmonary trunk, and ductus arteriosus. The coronary system is supplied retrogradely with oxygenated blood via the hypoplastic ascending aorta. Frequently there is associated coarctation at the isthmus.

\section{Proposed surgical solution}

The purpose of the suggested operation is to create separate circulations, enabling the systemic venous blood to flow into the lung only and the pulmonary venous blood to flow into the aorta. The procedure would use cardiopulmonary bypass, deep hypothermia, cardioplegia, and total circulatory arrest. The arterial connexion of the patient to the cardiopulmonary bypass machine would be via the iliac artery, provided the descending thoracic aorta is normal. The venous drainage would be via the iliac veins and temporarily also via the right atrium by cannulation of both superior and inferior venae cavae. At the same time as the iliac cannulation was being carried out, a midline sternotomy would be performed, the pericardium being opened laterally to permit its use later as patching material. Tourniquets would be placed around the venae cavae and purse string sutures placed in the right atrial appendage and antero-inferior wall of the atrium (fig 1) for venous cannulation. The patient would be cooled until rectal temperature reached $16^{\circ} \mathrm{C}$. The right atrium would be opened (fig 1) by creating a " $U$ " flap hinged posteriorly. By folding the flap into the atrium, the lumen created would be no smaller than the inferior vena cava, possibly larger. The interatrial septum would then be totally excised creating a large common atrium (fig 2). As the temperature reached $16^{\circ} \mathrm{C}$ 


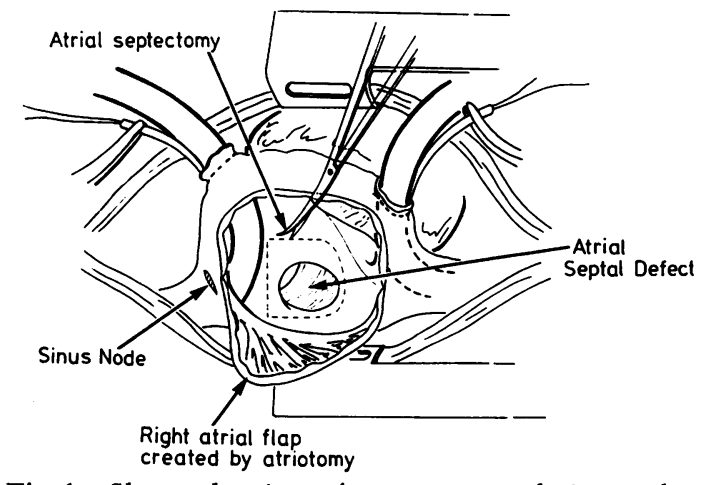

Fig 1 Shows the sites of venous cannulation and the line of atriotomy creating a right atrial flap. The sinus node is preserved. A complete atrial septectomy is carried out along the dotted line.

the extracorporeal circulation would be stopped, all aortic branches cross-clamped and cardioplegic solution injected via a polyethylene catheter, introduced retrogradely into the ascending aorta. After removal of the cannulae, the infolded right atrial flap would be sutured with continuous suture material along the dotted line shown in fig 2, taking care to avoid the sinus node, so as to create a long tunnel with no opening at the atrial level but markedly enlarging the residual left atrium (fig 3 ). A longitudinal incision would now be made in the anterior wall of the superior vena cava somewhat larger than the diameter of the underlying right pulmonary artery but at the level where these vessels cross

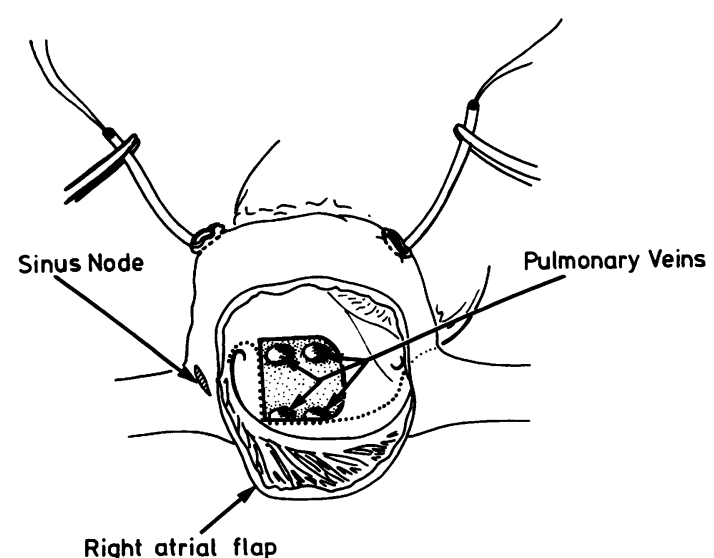

Fig 2 Shows the right atrium after removal of both superior and inferior caval cannulae. The dotted line represents the suture line of the enfolded right atrial flap avoiding the sinus node. The four pulmonary veins are seen.

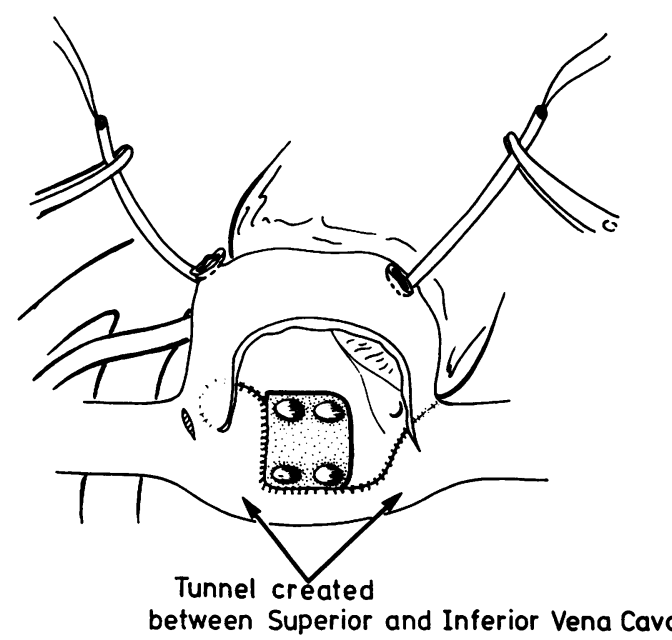

Fig 3 Shows the newly created continuity of inferior vena cava and the superior vena cava after the suturing of the right atrial flap has been completed.

A "window" could then be created between the posterior wall of the superior vena cava and then right pulmonary artery using simple stitcheso (fig 4). The window would be made as large as possible, producing no kinks in the pulmonaryô

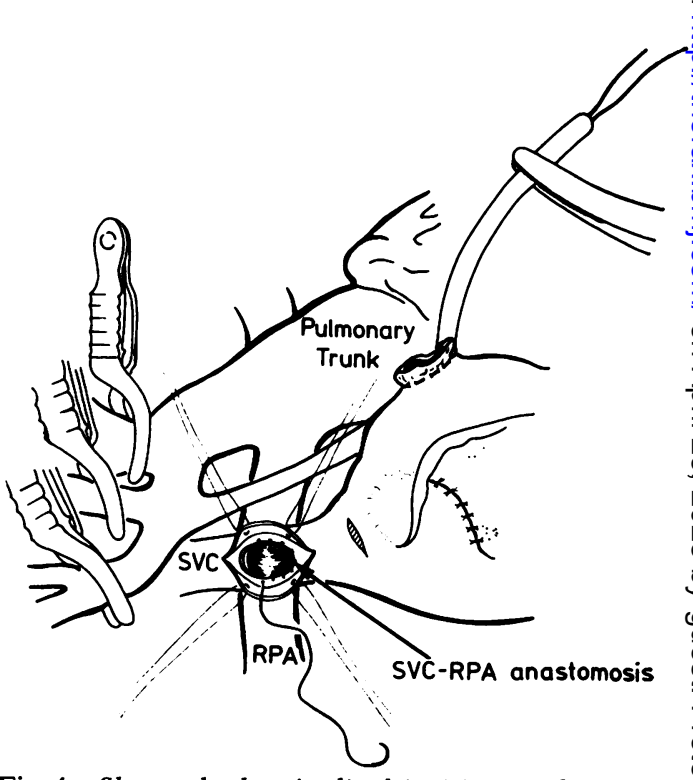

Fig 4 Shows the longitudinal incision in the anterio

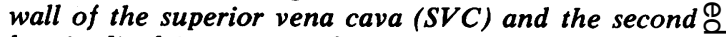
longitudinal incision in the posterior SVC wall.

Note the side-to-side anastomosis between the SVC and the right pulmonary artery (RPA). 


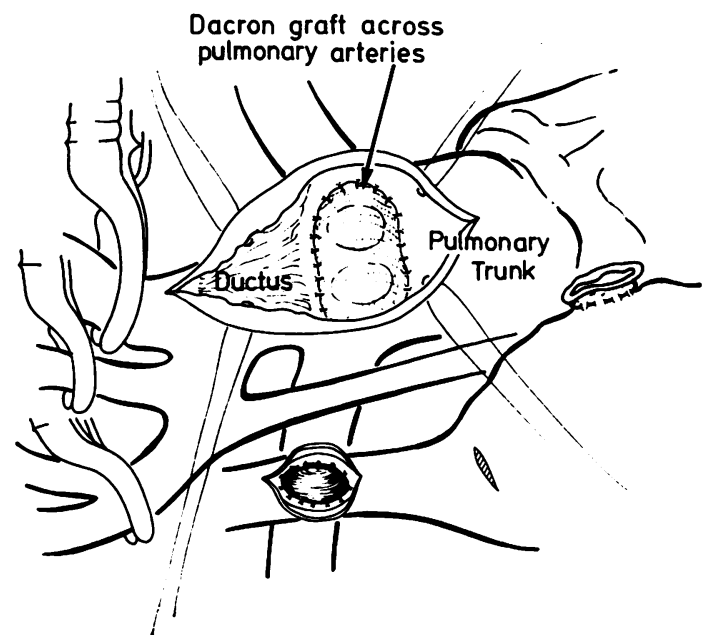

Fig 5 Shows the incision extending from the main pulmonary artery through the ductus arteriosus into the aorta. Note dacron graft "covering" and linking the right and left pulmonary arteries.

artery, so as to allow blood to flow freely in both directions. A longitudinal incision would then be made from the base of the main pulmonary artery through the patent ductus arteriosus until normal aortic tissue was reached. In order to link the flow between the right and left pulmonary arteries in an optimal fashion, one may choose one of the following techniques: (a) primary anastomosis in an end-to-end fashion using simple stitches; (b) patch graft angioplasty; (c) resection from the main pulmonary artery and end-to-end anastomosis.

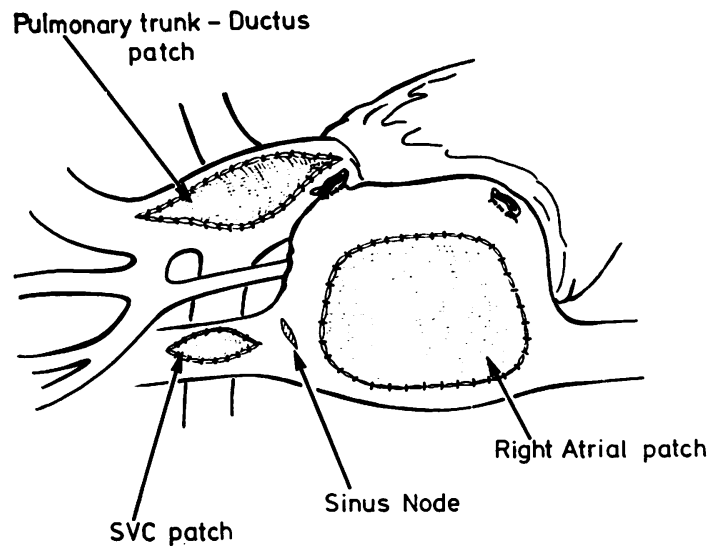

Fig 6 The completed operation showing the patch closure of the three surgically created "defects".
After the completion of the anastomosis of both pulmonary arteries (fig 5), all remaining defects. namely a large right atrial defect, a superior vena caval defect, and the main pulmonary artery-ductus-aortic defects would be closed with pericardium or synthetic material as preferred (fig 6). The extracorporeal circulation would be restarted, clamps and tourniquets removed, air evacuated from the heart, the patient rewarmed, the heart defibrillated and at adequate temperature taken off bypass.

\section{Comments}

The morphology created by this suggested operation would direct the entire systemic venous return through the superior vena cava to the right pulmonary artery then to both lungs. The pulmonary venous return would pass into an enlarged "left" atrium, consisting of the original left atrium and the atrioventricular vestibule of the right atrium. It would flow through the tricuspid valve, into the right ventricle and exit through the pulmonary trunk which would then supply the aortic arch and its branches.

For the operation to be successful, the pulmonary vascular resistance would need to be low. The resection of the interatrial septum and enlargement of the new "left" atrium would contribute to lowering of the pulmonary vascular resistance on the atrial side.

Avoiding the sinus node and atrioventricular nodes when the right atrial tunnel was created, would permit the heart to function in sinus rhythm, and permit use to be made of persisting atrial contraction. In the presence of a valveless right-sided heart it would seem reasonable to assume the need for a relatively high central venous pressure in the immediate postoperative course. ${ }^{4}$ Obviously, the superior vena cava-right pulmonary artery anastomosis should be created as wide as possible so as to allow all the systemic venous blood to enter the pulmonary system and obviate as far as possible any venous obstruction. The suggested operation, if successful, should enable the patient to grow, since it would not be limited by fixed sizes of various grafts and conduits as would occur if a previously suggested "corrective" operation was employed in treatment of this otherwise lethal lesion. ${ }^{3}$

We are indebted to Miss Noelle de Freitas for her help in the preparation of the manuscript. RHA is supported by the Joseph Levy Foundation together with the British Heart Foundation. 


\section{References}

1 Redo SF, Engle MA, Ehlers KH, Farnworth PB. Palliative surgery for mitral atresia. Arch Surg 1967; 95:717-23.

2 Brockman JL. Congenital mitral atresia. Am

Heart $J$ 1950; 40:301-5.

3 Albert HM, Bryant LR. A proposed technique J Cardiovasc Surg 1978; 19:257-60.

4 Fontan F, Baudet E. Surgical repair of tricuspi atresia. Thorax 1971; 26:240-8. for treatment of hypoplastic left heart syndrome 